\title{
Informationsgesellschaft und flexiblere Arbeitsmärkte
}

\author{
Gunther Tichy
}


(ITA-02-03)

\title{
Informationsgesellschaft und flexiblere Arbeitsmärkte
}

\section{Gunther Tichy}

\section{Keywords}

Informationsgesellschaft, Arbeitsmarkt, Flexibilität, Komplexität, firmenspezifisches Humankapital

\begin{abstract}
It is widely believed that the Information Society and its economic counterpart, the New Economy, require more flexibility especially in the labour market (i. e. less long term contracts) to allow faster reactions to the increased speed of change. The paper argues that increased complexity rather than faster change is the real problem. Dealing with complexity requires adaptability and organisational learning. Short term flexibility, however, is detrimental to adaptability and learning as it hinders the accumulation of firm-specific capabilities - and country-specific ones on the macro level. The ability of the firm to absorb and act upon knowledge depends to a large extent on staff continuity.
\end{abstract}

(c) IT_ - Institut für Technikfolgen-Abschätzung 


\section{Inhalt}

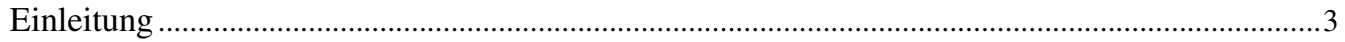

1 Die unzureichende Begründung des Bedarfs an größerer Flexibilität...........................................

2 Die tieferliegenden Ursachen des Suchens nach neuen Konzepten................................................6

3 Die mangelnde Berücksichtigung von Komplexität in den traditionellen

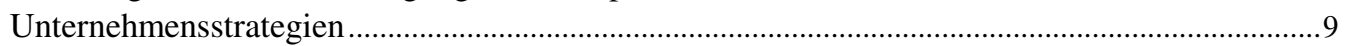

4 Ansatzpunkte einer Strategie des Umgangs mit Komplexität ......................................................12

4.1 Elastizität und Anpassungsfähigkeit als institutionelle Voraussetzungen......................................12

4.2 Corporate Vision und organisatorisches Lernen als Instrumente.................................................14

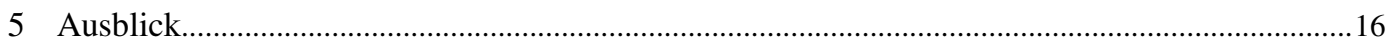

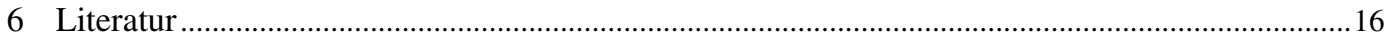




\section{Einleitung}

Kein Schlagwort prägt unsere Zeit mehr als das von der Informations- oder gar Wissensgesellschaft, ökonomisch realisiert in der ,New Economy'. Die sozialwissenschaftlichen Journale untersuchen diese neuen Begriffe nach allen denkbaren Kriterien, OECD und EU sehen in dem raschen Übergang zur Informationsgesellschaft die einzige Chance Europas, den amerikanischen Wachstumsvorsprung aufzuholen, Politiker aller Couleurs und Massenmedien versprechen sich von der New Economy rasches, inflations- und rezessionsfreies Wachstum. Allerdings bedürfe es für den Übergang in die neue Welt mehr Flexibilität, vor allem auf dem Arbeitsmarkt. Der deutsche Sachverständigenrat fasst das unter dem Titel: „Anpassungen und Chancen in der Neuen Ökonomie“ klar zusammen: „Die Vorstellung von Arbeit als räumlich und zeitlich festgelegte, kontinuierlich abzuleistende Erwerbstätigkeit verliert an Bedeutung. ... [Es] werden sich zunehmend Beschäftigungsformen etablieren, die jenseits des traditionellen Arbeitsvertrags stehen." Jeder Arbeitnehmer werde sich auf mehrmaligen Berufswechsel im Laufe seines Lebens einstellen müssen (Peters 1994; Handy et al 1997; Föhrenbergkreis 1997). Das ist eine völlig neue Vorstellung von Flexibilität; Hammermesh (1993) betont, dass früher "... labor market flexibility has meant the ability to adjust labor costs and employment conditions to improve economic efficiency. In recent times the demand for flexibility has translated into a demand for new work practices and patterns, which aim to increase physical and functional mobility of labor and to relax job security regulations."

Was bedingt diesen radikalen Bruch mit der Vergangenheit, in der - ganz im Gegenteil - gerade die langjährige Betriebszugehörigkeit honoriert und gefeiert wurde? Im folgenden wird versucht, von den oberflächlichen Begründungen des Gesinnungswandels ausgehend, dessen tieferliegende Ursachen herauszuarbeiten: zunehmende Komplexität scheint die wichtigste unter ihnen zu sein. Flexibilisierung jedoch vergrößert Komplexität anstatt einen Beitrag zu ihrer Überwindung zu leisten. Demgemäss wird im weiteren nach adäquaten Strategien zur Bewältigung der zunehmenden Komplexität gefragt. 


\section{Die unzureichende Begründung des Bedarfs an größerer Flexibilität}

Die Promotoren größerer Arbeitsmarktflexibilität stellen stets dieselben Versatzstücke ins Zentrum: Unsere Welt sei raschlebiger, dynamischer geworden, der technische Fortschritt rascher, die Produktlebenszyklen kürzer, die Konkurrenz härter. Man müsse auf unvorhergesehene Ereignisse rascher reagieren können, längerfristige Konzepte würden angesichts der Dynamik immer schneller obsolet. Aus der , digitalen Revolution“ resultiere ein völlig neues Paradigma. Die günstige Entwicklung der amerikanischen Wirtschaft im letzten Jahrzehnt zeige die wohltuende Wirkung von Flexibilität und Deregulierung. Als Idealbild der Zukunft wird die virtuelle Firma gesehen, die nur aus einer Idee besteht, die extern, von ad hoc zusammengestellten Spezialistenteams entwickelt, extern, vom jeweiligen Billigstbieter produziert, und extern, über selbständige Firmen vertrieben wird; eine solche Konzeption entspräche auch voll dem Marktmodell der (neo)klassischen Theorie, in dem marktmäßige Suchprozesse die jeweils optimalen Anbieter von Produktionsfaktoren aufspüren, somit zu optimaler allokativer Effizienz führen.

Vieles spricht dafür, dass die meisten dieser Argumente eher den Zeitgeist spiegeln als einen tatsächlichen Bruch mit der Vergangenheit. Soweit sich aber ein derartiger Bruch tatsächlich nachweisen ließe, ist zu fragen, ob und in welchem Maße höhere Arbeitsmarktflexibilität die einzige oder wenigstens die entscheidende Gegenstrategie wäre.

Dass die Zeit ,raschlebiger ' geworden ist, lässt sich natürlich schwer messen, und die Einschätzung dürfte auch stark von subjektiven Elementen geprägt sein. Was an Evidenz vorliegt spricht nicht unbedingt in diese Richtung: Die vielzitierte ,elektronische Revolution' etwa scheint keineswegs rascher verlaufen zu sein als etwa die ,Verkehrsrevolution' durch Erfindung der Dampflokomotive. ${ }^{1}$ Auch der wichtigste messbare Indikator des ,Tempos' des , technischen Fortschritts', die totale Faktorproduktivität, lässt keine Beschleunigung erkennen: Ganz im Gegenteil hat sich der technische Fortschritt in Europa nach diesem Indikator in den neunziger Jahren sogar deutlich verlangsamt; in den USA hat er sich zwar beschleunigt, doch ausgehend von dem sehr niedrigen Tempo der siebziger und achtziger Jahre, sodass er noch immer langsamer ist als in Europa (Scarpetta et al 2000) ${ }^{2}$ das relativiert auch die beliebte monokausale Erklärung des amerikanischen Beschäftigungswunders als Folge der digitalen Revolution der New Economy. Ebenso skeptisch sollte die behauptete Beschleunigung des Produktzyklus gesehen werden. Zwar gibt es durchaus Bereiche, in denen grundlegende Innovationen rasche Änderungen des Produktangebots erzwingen; in den meisten Fällen handelt es sich bei den ,neuen ' Produkten jedoch um marginale Änderungen, die eher Marketingüberlegungen folgen und kaum umfangreiche Änderungen der Produktions- und Vertriebssysteme erfordern.

Auch der zweite behauptete Bruch, der angebliche Übergang zur New Economy als völlig neuem Paradigma ist zu hinterfragen: Manche der neueren Untersuchungen schätzen die Folgen der Informationstechnologie als deutlich weniger revolutionär ein als die Erfindungen in der zweiten Hälfte des 19. Jahrhunderts (Gordon 2000); dieser Eindruck verstärkt sich, wenn man nicht bloß die technischen Innovationen des Elektronikzeitalters mit früheren vergleicht, sondern auch die organisatorischen (z. B. Fordismus, Divisionalisierung, Versandhandel). Zunehmend wird betont, dass auch die New Economy den alten Gesetzen der Ökonomie gehorche: "Ignore basic economic prin-

1 Von der Konstruktion der ersten funktionierenden Dampflokomotive durch Stephenson im Jahre 1814 dauerte es 60 Jahre bis zwei Drittel des gesamten englischen Eisenbahnnetzes errichtet waren (nach 70 Jahren waren wehr als vier Fünftel errichet); seit der Verfügbarkeit der ersten funktionsfähigen Computer (ZUSE Z4 1945, ENIAC 1946) sind immerhin schon 56 Jahre vergangen.

2 Alle geschätzten Varianten der totalen Faktorproduktivität zeigen diese Tendenz. 
ciples at your own risk. Technology changes. Economic laws do not." (Shapiro and Varian 1998). Die Sekundärfolgen der Digitalisierung auf die anderen Branchen, insbesondere deren Organisation und Beschäftigung, liegen noch vor uns; die zunehmende, Elektronisierung ' wird sicherlich zu zunehmender Komplexität beitragen.

Selbst wenn wir jedoch in einer neuen Welt lebten: könnte eine weitgehende Flexibilisierung der Arbeitsverhältnisse und weitgehendes Outsourcing - beide Übergang von hierarchischen (firmeninternen) Mechanismen (Organisation) auf Marktbeziehungen - die Allokation tatsächlich verbessern. Das Argument muss sich einerseits der Konfrontation mit der historischen Entwicklung stellen, in der die Unternehmensgröße im Zeitverlauf markant zunahm, bei gleichzeitig zunehmend komplexer werdenden Wirtschaftsbeziehungen, der Anteil der Markttransaktionen mit steigender Komplexität also abnahm. Andererseits gilt es zu berücksichtigen, dass Effizienz von Markttransaktionen volle Transparenz der Information, vollständige Verträge und volle Appropriierbarkeit voraussetzt, Voraussetzungen die in der realen Welt eher selten vorliegen; die Existenz von Unternehmen, also der Ersatz von Markttransaktionen durch Organisation, basiert auf Unsicherheit, unvollständiger und asymmetrischer Information, daraus folgend unvollständigen Verträgen, steigenden Skalenerträgen und Spezialisierungsvorteilen. Die Theorie des Unternehmens ${ }^{3}$ zeigt, dass hierarchische Organisation marktmäßiger stets dann überlegen ist, wenn es um spezialisierte Produktionsmittel und seltene bzw. komplexe Transaktionen geht, ${ }^{4}$ der Erfolg der Tätigkeit nicht leicht zu bewerten ist, die Transaktionen eng miteinander verflochten sind, oder eine Weitergabe wichtiger Informationen unerwünscht ist. ${ }^{5}$ Alles das scheint in unserer Zeit an Bedeutung eher zu- als abzunehmen.

Überdies steht die Marktideologie der Flexibilisierungskonzepte auch in markantem Gegensatz zu dem tatsächlichen Verhalten der Unternehmen am Markt: Diese tendieren - vor allem im Wege von Fusionen - immer größer zu werden; die 'mission', das erklärte Ziel so gut wie aller Großunternehmungen besteht darin, auf ihrem jeweiligen Gebiet weltweit zu den größten Drei zu gehören. Eine solche Firmenstrategie führt unvermeidlich zu einer oligopolistischen Marktstruktur, und keineswegs zum Marktmodell, das perfekten Wettbewerb voraussetzt, eine Struktur also, in der jeder Marktteilnehmer so klein ist, dass er den Preis nicht beeinflussen kann.

Der vermutete unmittelbare Zusammenhang zwischen Flexibilisierung und Effizienz wie die Betonung der Effizienz als alles überragendem betrieblichen und gesamtwirtschaftlichen Maßstab übersehen, dass Effizienz zahlreiche, zumeist langfristige Bestimmungsgründe hat. Sie reichen vom Betriebsklima und der Akkumulation unternehmensspezifischer Fähigkeiten über die Bildung entsprechender Netzwerke bis zu einem gewissen Maß an Redundanz, das es ermöglicht, über die unmittelbare Unternehmensperspektive hinauszublicken. Die Effizienzsteigerungskonzepte zahlreicher Unternehmensberater und Manager übersehen, dass kurzfristige Effizienzsteigerung langfristige vielfach ausschließt. Swann $(1998,109)$ spricht in diesem Zusammenhang von "corporate myopia", und leider lässt sich inzwischen auch eine 'policy myopia', eine volkswirtschaftliche Kurzsichtigkeit erkennen, die gefährliche Übertragung - zum Teil nicht unbedenklicher - betriebswirtschaftlicher Konzepte auf die Volkswirtschaft.

3 Siehe etwa Holmstrom and Tirole 1989 oder Milgrom and Roberts 1992.

4 Ein Zulieferer wird nicht bereit sein, teure Investitionen zu tätigen, die bloß für einen Kunden genützt werden können, da er damit rechnen muß, nach der Investition einem erheblichen Preisdruck seines Kunden ausgesetzt zu sein ('ex-post opportunism').

5 Etwa bei der Entwicklung neuer Produkte. Effiziente Märkte kann es bloß dort geben, wo eine entsprechend große Zahl von Käufern und Verkäufern dasselbe - standardisierte - Produkt nachfragt; je geringer deren Zahl bzw. deren Nachfrage, d. h. je spezifischer das Produkt, desto dünner der Markt, desto höher die Suchkosten für die Beteiligten, desto ineffizienter der Markt, selbst wenn es ihn gibt. 


\section{Die tieferliegenden Ursachen des Suchens nach neuen Konzepten}

Die beschränkte Überzeugungskraft der gängigen Argumente zugunsten einer stärkeren Flexibilisierung wirft die Frage auf, ob die diesbezüglichen Forderungen von OECD, EU, Regierungen, Unternehmern und Unternehmensberatern auf falschen Vorstellungen beruhen. Im Folgenden wird die These aufgestellt, dass Europa zwar tatsächlich vor neuen Problemen steht, doch solchen die tiefer liegen; sie erfordern grundsätzlichere Reformen, Flexibilisierung als solche würde die Probleme eher verschärfen. Die Ansatzpunkte zum Verständnis der wirklich neuen Probleme sollen im Folgenden kurz charakterisiert werden:

Zwar keineswegs neu, aber deutlich stärker ausgeprägt als früher, ist die zunehmende Spezialisierung der Information, die laufend neue Fachgebiete entstehen lässt ${ }^{6}$ und Kooperationen und Netzwerke erzwingt. ${ }^{7}$ Tätigkeiten werden ausgelagert, weil die Spezialisten, in Spezialfirmen konzentriert, ihr Spezialwissen vertiefen und Größenvorteile nutzen, somit effizienter eingesetzt werden können. Der intellektuelle Taylorismus, die radikale Arbeitsteilung auch im Bereich der geistigen Arbeit, die die Informationsgesellschaft zunehmend erzwingt, erfordert nicht bloß Interdisziplinarität sondern auch Kooperation auf höherer Ebene, ein Erfordernis, das die Komplexität erhöht und die Beteiligten vielfach überfordert. ${ }^{8}$

Die zunehmende Spezialisierung in der Informationsgesellschaft ergibt sich zunächst aus dem kumulativen Charakter des Wissens. Obwohl neue Information alte vielfach obsolet oder trivial macht, wächst die gesamte Informationsmenge laufend an; sie muss nicht bloß gelernt und in Evidenz gehalten, sie müsste auch reflektiert werden. Der, elektronische Ausweg': zwar nicht die Fakten zu wissen, wohl aber die Methode, wie und wo sie gesucht werden können, führt bestenfalls zu strukturierten Informationen, keinesfalls aber zu reflektiertem Wissen. Je schmaler jedoch das Teilgebiet der Spezialisierung, auf dem der Spezialist über reflektiertes Wissen verfügt, desto weniger kann er Ergebnisse auf breiteren und relevanten Gebieten erarbeiten; desto mehr ist er auf die Kooperation mit anderen Spezialisten angewiesen - mit allen Komplexitätsproblemen der interdisziplinären Zusammenarbeit.

Die zunehmende Spezialisierung des Wissens resultiert weiters aus der zunehmenden Differenzierung der Aufgaben. ${ }^{9}$ Kein Unternehmen kommt mehr mit Kenntnissen einfacher Mechanik aus; elektronische Steuerung, Mess- und Regeltechnik, Simulation, etc. erfordern jedoch nicht bloß zusätz-

6 Manche ,Spezialisierung', nicht zuletzt im Bereich der Wissenschaft entspricht allerdings eher dem krampfhaften Versuch einer Produktdifferenzierung zwecks Verbesserung der, Marktchancen“

7 Wenn die meisten Komponenten eines Kraftfahrzeugs heute von Spezialisten zugeliefert werden, ist das weniger Rationalisierung als Spezialisierung: das Spezialwissen für Entwicklung und Erzeugung ist in der Automobilfabrik nicht vorhanden, und zu vertretbaren Kosten nicht erwerbbar. Gleiches gilt für den administrativen und organisatorischen Bereich, wo EDV-Spezialisten, Kostenrechnungs-Spezialisten, SteuerSpezialisten, Organisations-Spezialisten erforderlich geworden sind.

8 Der intellektuelle Taylorismus wirft darüber hinaus durchaus ähnliche Probleme auf wie der Taylorismus im manuellen Bereich: Erschwerung der Reflexion, Verlust des Verständnisses der Zusammenhänge, potentiell rasche Entwertung der Spezialisierung durch neues Spezialwissen auf anderen Gebieten, usw. Die Diskussion über Lebenslanges Lernen spiegelt genau diesen Aspekt rascher Veralterung allzu engen Spezialwissens.

9 Ein einfacher robuster Motor kann von wenig spezialisierten Ingenieuren leicht und rasch entwickelt werden, und manche Entwicklungsländer (etwa China) nutzen das auch. Einen Automotor zu entwickeln, der zugleich sparsam, abgasarm, vibrationsfrei, leise und dauerhaft ist, bedeutet hingegen eine Optimierungsaufgabe, einen Aufwand in Milliardenhöhe und einen Spezialisierungsgrad, der allein von einigen wenigen spezialisierten Entwicklungsfirmen geleistet werden kann. 
liche Spezialkenntnisse, sondern vielfach auch deren Kombination. ${ }^{10}$ Die Technik ist unsichtbar und komplizierter, komplexer ${ }^{11}$ und Spezialisten-abhängiger geworden, der Spezialist Technik-abhängiger.

Über die technische Komplexität hinaus hat auch die organisatorisch-führungstechnische Komplexität zugenommen. Der zunehmende Kostendruck zwingt die Unternehmen zu immer raffinierteren Rationalisierungsmaßnahmen im Bereich der Sicherung der Kapazitätsauslastung (Flexibilität), der Zulieferung, der Logistik und des Vertriebs; es müssen komplexe organisations- oder informationstechnische Instrumente eingesetzt werden, die allein von Spezialisten entwickelt und betreut werden können. Die Informationstechnik ermöglicht es dem Marketing, die spezifischen Wünsche des Kunden immer genauer zu kennen, wirft damit aber zugleich auch komplexe Entscheidungsprobleme auf, in Bezug auf Produktionsanpassung wie Logistik. Auch die rasant zunehmende größenordnungsmäßige und geographische Dimension der Firmen und Firmenkonglomerate vergrößert die Komplexität effizienter Führung; sie erfordert zunehmend komplexere Instrumente.

Ähnlich wie die Aufgaben der Firmen haben sich auch die Bedürfnisse der Kunden zunehmend differenziert. ${ }^{12}$ Mit zunehmendem Wohlstand haben Erfahrungsgüter, deren Qualität erst durch längeren Gebrauch erkannt werden kann (gegenüber Verbrauchsgütern) an Bedeutung gewonnen. Dennoch ändern die Firmen im Kampf um Marktanteile ihre Produkte relativ häufig - wenn auch zumeist keineswegs in grundlegender Weise - und vergrößern damit die Unsicherheit der Kunden; die Marktreaktionen treten verzögert auf und werden erratischer.

Alle diese Entwicklungen wurden durch die Mikroelektronik (im weitesten Sinn) erheblich verstärkt. Zuvor war die standardisierte Massenproduktion, die primär den Preis als Wettbewerbsinstrument einsetzte (Großindustrie - Fordismus), von der qualitativ hochwertigen, maßgeschneiderten Einzelund Kleinserienproduktion (Kleinindustrie - Handwerkstechnologie) deutlich separiert. Die Mikroelektronik ermöglichte auch der Großindustrie diversifizierte, qualitativ hochwertige Kleinserienproduktion. Damit wurde einerseits der Wettbewerb schärfer, andererseits mussten alle Unternehmen - d. h. ihre Belegschaft - zusätzliche Kompetenzen in Elektronik wie in neuen Formen der Unternehmensorganisation und der Logistik erwerben. Der Komplexitätsgrad stieg beträchtlich an.

Die Informationsintensität der Wirtschaftsprozesse ist weiters durch die Verwissenschaftlichung der Innovation gestiegen. ${ }^{13}$ Statt des linearen Innovationsprozesses - von der Invention aus weitgehend autonomer Forschung oder Zufall über die Innovation zur Produktion - erzwingt der verstärkte Wettbewerb die systematische und laufende Innovation: Wissenschafter wie Firmen forschen unmittelbar anwendungsorientiert; vielfach lässt sich Grundlagenforschung von Umsetzung nicht mehr unterscheiden. ${ }^{14}$ Wissen, und zwar in höchstem Maße spezialisiertes Wissen, ist in diesem

${ }^{10}$ Das gilt selbst für die einfache Autoreparatur, bei der die Inspektion durch den Mechaniker unter der Hebebühne durch den Blick des Spezialisten auf das elektronische Testgerät ersetzt wurde.

11 Kompliziertheit ist der Grad der Unterschiedlichkeit einzelner Elemente des Systems, Komplexität die Art und Zahl der Relationen zwischen den Elementen (G. Klaus, 1969, Wörterbuch der Kybernetik).

12 Ford konnte es sich noch leisten, sein Modell T allein in Schwarz anzubieten, Volkswagen konnte mit einem einzigen, im Zeitverlauf bloß marginal veränderten Modell jahrzehntelang Marktführer bleiben. Der uniformierte Massenindividualismus unserer Tage erfordert unzählige Varianten desselben Massenmodells (im Kfz-Bereich ,Plattform-Strategie'), die höchst spezialisierte Anforderungen an die (flexible) Massenproduktion und, mehr noch, an die Logistik stellen.

13 Auch das ist nicht neu: Daß die Europäer irgendwann im angeblich dunklen Mittelalter „das Erfinden erfunden" haben (Landes 1999, 61), ist eine der zentralen Ursachen ihres heutigen Wohlstands und des Überholens der viel älteren (und zunächst auch wohlhabenderen) Zivilisationen Asiens.

14 Bei den ,star scientists ' antichambrieren die Vorstandsdirektoren mit Forschungsaufträgen und die VentureKapitalisten mit Geld; man gründet Firmen, , start-ups', lange bevor die Idee marktreif ist - hier ist die immer wieder behauptete Beschleunigung, die beim technischen Fortschritt keineswegs zu erkennen ist, sehr wohl Realität. Beschleunigt hat sich allerdings vor allem der Run auf die neue Idee; zu neuen Produkten kommt es zumeist auch nicht rascher, wie das Beispiel der Gentechnik oder die jüngste Krise der dot.com-Firmen illustrieren. 
Prozess der kontinuierlichen und forcierten Suche nach neuen Ideen und ihrer Umsetzung von höchster Bedeutung, und zwar nicht bloß auf Seite der Wissenschafter, sondern mindestens ebenso sehr auf Seite der Auftraggeber und der Financiers. Innovation, und mehr noch die Fähigkeit kontinuierlich Sequenzen miteinander verbundener, synergistischer Innovationen hervorzubringen, ist eine der schwierigsten Unternehmeraufgaben, eine Aufgabe von hohem Komplexitätsgrad.

Schließlich sind auch noch die Rückkopplungseffekte zu beachten: Wenn sich ein Wirtschaftssubjekt in einer interdependenten Welt zu Änderungen seines Verhaltens gezwungen sieht, ändert das die Umweltbedingungen für die anderen und zwingt diese zu Reaktionen. In einer komplexen Welt ist jedoch nicht bloß eine einzige, für die anderen abschätzbare Reaktion möglich, sondern unzählige. Daraus entsteht Unsicherheit der Betroffenen, die diese zu einer Anpassung ihrer Strategie in einer Form zwingt, die ihrerseits viele Reaktionsmöglichkeiten offen hält, woraus weitere Komplexität für die dadurch Betroffenen entsteht: "Increasing complexity feeds on itself, generating more complexity in the process." (Trisoglio 1995, 8).

Alle diese Argumente bedingen größere Komplexität im Sinn zunehmender Art und Zahl der Relationen zwischen den Elementen des Systems; sie bedingen, dass sich Phänomene nicht mehr auf einfache Ursachen zurückführen lassen, Nicht-Linearitäten an Bedeutung gewinnen, und Daten der Vergangenheit nur begrenzt Schlussfolgerungen für Gegenwart und Zukunft erlauben. Perfekte Anpassung an eine stabile prognostizierbare Entwicklung ist jedoch die Strategie, die die Firmen in der weitgehend strukturkonstanten Vergangenheit verinnerlicht haben. Da perfekte Anpassungsleistung i.d.R. bloß um den Preis verminderter Anpassungsfähigkeit zu erlangen ist, mehren sich die Probleme bei ,Störungen' und ,Flexibilisierung' erscheint erforderlich. Die Flexibilisierung von Unternehmensstrukturen und Arbeitsverhältnissen trägt jedoch selbst zur weiteren Komplexität bei, sodass die Lösung in einer weiter gehenden Systemreform gesucht werden muss: Komplexe Systeme ,müssen mit internen Unwahrscheinlichkeiten und Unzulänglichkeiten zurechtkommen. Sie müssen Einrichtungen entwickeln, die genau darauf aufbauen, etwa Einrichtungen, die abweichendes Verhalten reduzieren, das erst dadurch möglich wird, dass es dominierende Grundstrukturen gibt. Komplexe Systeme sind mithin zur Selbstanpassung gezwungen, und zwar im Doppelsinne einer eigenen Anpassung an die eigene Komplexität. Nur so ist zu erklären, dass Systeme den Veränderungen ihrer Umwelt nicht bruchlos folgen können, sondern auch andere Gesichtspunkte der Anpassung berücksichtigen müssen.“ (Luhmann 1988, 56).

Das wirklich Neue ist somit die gestiegene Komplexität, der sich Manager wie auch Politiker gegenüber sehen und - als unmittelbare Reaktion darauf - eine generelle Tendenz zu kurzfristiger Perspektive. Diese neue Komplexität hat nichts mit dem Tempo des Wandels zu tun; auch rascher Wandel ist beherrschbar, wenn die Richtung der Änderung vorhersehbar ist. Komplexität bedeutet jedoch, dass die Richtung der Änderungen nicht ohne weiteres vorhersehbar ist, und dass mögliche Alternativen nicht leicht auf eine gemeinsame Basis gebracht werden können; es gilt nicht bloß Risken abzuwägen, es geht um Entscheidungen unter Unsicherheit. Die aus dem Übergang zu netzwerkartigen Strukturen resultierende, winner takes it all'-Gefahr verschärft das Entscheidungsproblem weiter. Die Reaktion, sich unter diesen Voraussetzungen alle Möglichkeiten offen zu halten, möglichst große Flexibilität zu schaffen, ist nur zu verständlich. 


\section{Die mangelnde Berücksichtigung von Komplexität in den traditionellen Unternehmensstrategien}

Zunehmende Komplexität erschwert oder verunmöglicht Art und selbst Richtung möglicher Änderungen zu prognostizieren, verhindert demgemäß längerfristige Planung traditioneller Art (Marktstudien und Prognosen) und macht die klassischen Managementinstrumente, wie MBO (Management by objectives) oder PPBS (Planning, programming, budgeting systems) obsolet, die alle von einer linearen, mechanistischen Entwicklung ausgehen. Für sie ist Management ein Prozess der Planung, Organisation, Koordinierung, Anordnung und Kontrolle; ihr Ziel ist Effizienz und Optimierung. In einer komplexen, vernetzten Welt stehen Effizienz und Optimierung zweitrangig hinter der Entwicklung der richtigen Strategie, die die entsprechenden technologischen und Markttrends nutzt. "The appropriate motto is 'Do the right thing', rather than 'do things right'." (Trisoglio 1995, 22). Was heißt aber "do the right things"? Orientierung an den Kundenwünschen, Wertanalyse, Maximierung des Shareholder value und Flexibilisierung dürften die derzeit am häufigsten empfohlenen Varianten sein. Sie alle bieten jedoch keine Lösung des Komplexitätsproblems.

Die vor allem in Europa zunehmend beliebte Strategie der strikten Orientierung an den Kundenwünschen reduziert zwar Komplexität, indem sie - passiv reagierend - das Problem auf die Kunden abschiebt: Diese wüssten selbst am besten, wie sich ihre Märke entwickeln und eine gute $\mathrm{Zu}$ kunft der Kunden bedeute auch eine gute eigene Zukunft. Insoweit stellen Kundenorientierung und gemeinsame Entwicklungsarbeit eine wichtige und auf mittlere Sicht unverzichtbare Strategie dar; sie bedarf aber einer ergänzenden Strategie mit langfristiger Perspektive. Einerseits können Kunden gar nicht wissen was sie wollen, wenn sie die neuen Möglichkeiten nicht kennen; ${ }^{15}$ andererseits unterliegt die Entwicklungszusammenarbeit mit den Kunden der Gefahr des Nutzer-Paradoxons (Tichy 2001), der Vernachlässigung weiterführender, radikaler Innovationen zugunsten inkrementaler: ${ }^{16}$ Die Kunden sind primär an Verbesserungen ihrer Produkte interessiert, wirklich neue Ansätze kommen in aller Regel von neuen Firmen, ${ }^{17}$ also von Firmen, die noch gar nicht Kunden sein können.

Konzepte wie Wertanalyse oder Return on Investment (ROI) liefern bei zunehmender Komplexität sogar irreführende Ergebnisse. Sie vernachlässigen gerade die zentralen Ursachen der Komplexität: Verbundeffekte, Synergien, und intertemporale Interdependenz; es gelingt diesen Konzepten nicht das Unternehmen als komplexes System zu verstehen. ${ }^{18}$

Das Versagen der traditionellen Managementkonzepte bei steigender Komplexität führte zu einer Forcierung der marktmäßigen Kontrolle der Unternehmensstrategien durch den Kapitalmarkt im Wege der Konzepte Shareholder Value und Market for Corporate Control: In den Marktentscheidungen über Kauf und Verkauf von Aktien konzentriere sich das gesamte Wissen aller Beteiligten, falsche Strategien der Firmen führten daher rasch zu sinkenden Kursen und entsprechendem Druck auf das Management; genüge das nicht, käme es im Wege des Market for Corporate Control zu

15 Ein beliebtes Beispiel dafür ist, daß die Damen kein Bedürfnis nach Seidenstrümpfen hatten, solange es diese nicht gab; in diesem Zusammenhang besser paßt wohl, daß edelstahlverarbeitende Kunden keine Ahnung davon haben, ob und wann Spezialkunststoffe verfügbar sein werden, die ihre Bedürfnisse besser erfüllen.

16 Christensen (1997) spricht in diesem Zusammenhang vom ,innovator's dilemma”.

17 Siehe dazu auch die Fallstudie über die Entwicklung der verschiedenen Generationen von Computer-Diskettensystemen von Christensen and Rosenbloom (1995).

18 Für eine genauere Darstellung siehe Schmidt und Maßmann 1999. 
einer feindlichen Übernahme und anschließender Reorganisation der Firma. Aber auch dieser Mechanismus zeigt keine Wege zur Lösung des Komplexitätsproblems auf. In der Theorie prämiert er diejenigen Firmen, die es gelöst haben, in der Praxis führt er zu kurzfristiger Disposition und damit zu Flexibilierungsstrategien. Der Ansatz setzt voraus, daß auf dem Aktienmarkt hochinformierte Personen agieren, die ihr eigenes Vermögen anlegen; tatsächlich jedoch fühlen sich die meisten Anleger zu wenig informiert und delegieren die Anlageentscheidung; fast alle am Aktienmarkt agierenden Personen legen daher fremdes Geld an, und sind in ein mehrgliedriges hierarchisches System eingebunden. Die Angestellten, die die Anlageentscheidungen treffen - Vermögensverwalter in den Banken oder Anlageverantwortliche in den Fonds - können Komplexität und langfristige Aspekte gar nicht berücksichtigen; sie müssen, wenn schon nicht vierteljährlich, so doch innerhalb von ein oder maximal zwei Jahren gegenüber ihren Vorgesetzten - und die Bank gegenüber ihren Kunden - Erfolge in Form von laufenden Erträgen nachweisen; ${ }^{19}$ die Kurzfristigkeit ist systemimmanent. ${ }^{20}$ Für komplexe Entscheidungen ist Ertragsmaximierung generell und erst recht kurzfristige Ertragsmaximierung ein Maßstab, der das Überleben gefährdet.

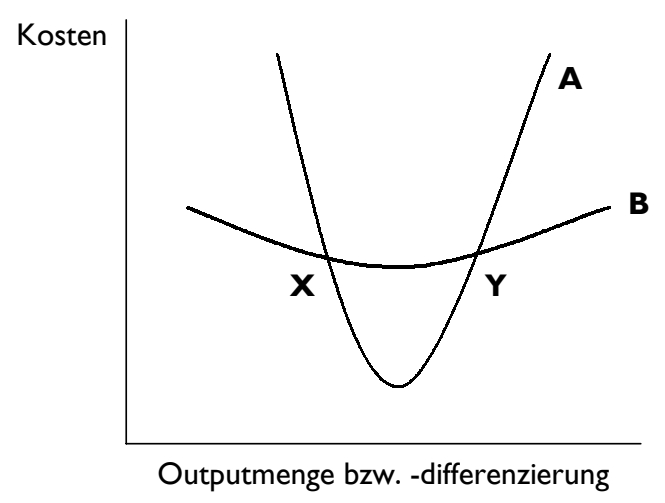

Abbildung 1: Outputflexibilität

Mangels anderer Instrumente erscheint Flexibilisierung aller Unternehmensbereiche somit unvermeidlich, um in einer nicht prognostizierbaren Welt auf Unvorhergesehenes reagieren zu können und zugleich auch der Kontrolle durch den Kapitalmarkt zu genügen. Allerdings besteht die Gefahr, dass Strukturen, die rasche Reaktionen ermöglichen (Flexibilisierung), die Anpassung an grundlegende Änderungen erschweren. Demgemäss erscheint es zweckmäßig, in der Tradition von

19 Die Banken und die Fonds müssen ihren Kunden wenigstens jährlich nachweisen, daß sie deren Geld zumindest so gut angelegt haben wie ihre Konkurrenten; sonst erleben sie die lange Sicht vermutlich nicht: "The big institutional investors nowadays increasingly react to short term pressure on investment performance ... (and) ... are unwilling to countenance long term investment" (Nigel Lawson, Chancellor of the Exchequer, zitiert nach Miles 1993). Der private Anleger mag sich einmal überzeugen lassen, daß der, relativ zur Konkurrenz unterdurchschnittliche Ertrag seines Portefeuilles im Sinne einer längerfristigen Ertragsmaximierung bewußt in Kauf genommen wurde; beim zweiten Mal wird er an der Anlagestrategie zweifeln, und zu den höher rentierenden Konkurrenten wechseln - eine Entscheidung, die unter Unsicherheit aus seiner Sicht nicht grundsätzlich falsch ist: Da die Zukunft unbekannt ist, kann der Anleger zwischen unfähigen Managern und langfristig schlechten Strategien schwer unterscheiden.

20 „Wer ständig den heißen Atem der Analysten im Nacken spürt und sich vom ihm jagen läßt, hat es schwer, eine längerfristige Strategie zu verfolgen. ... Vorstandschefs müssen heute ungleich mehr Zeit aufbringen für den Dialog, für die Kommunikation mit den Finanzmärkten. Sie sehen sich harten Fragen und Forderungen oft junger Analysten ausgesetzt, die gut ausgebildet und informiert sind. Die meisten haben allerdings ein Unternehmen noch nie von innen gesehen. Der CEO, der Unternehmensführer, mutiert so zum Chefverkäufer seines Unternehmens. Etwaige Zusatzqualifikationen als Entertainer schlagen sich nicht selten im Kurs nieder.“ (Schweickart 2001, 26). 
Klein (1984) zwei Arten von Flexibilität zu unterscheiden: Flexibilität I als Fähigkeit auf Änderungen (Risken) rasch zu reagieren, und Flexibilität II als Fähigkeit, neu auftretende Chancen nutzen zu können; bei ersterer geht es primär um Kosten, wie Abbildung 1 in Anlehnung an Stigler (1939) illustriert: Kurve A spiegelt eine Faktorenkombination (Technologie), mit der eine bestimmte, eng begrenzte Outputmenge oder -variation mit minimalen Kosten produziert werden kann; wird diese über- oder unterschritten, steigen die Kosten rasch an, die Flexibilität ist also gering. Kurve B spiegelt (bei gegebenen Preisen) eine Situation sehr viel größerer Flexibilität, zeigt allerdings auch die Kosten, mit der diese Flexibilität erkauft werden muss: Zwischen den Punkten X und Y produziert die flexible Technologie erheblich teurer. Was üblicherweise unter Flexibilisierung verstanden wird, entspricht Flexibilität I, dem Übergang von Kurve A zu Kurve B. Flexibilität II hingegen, als "possibility to move to various second period positions" (Jones and Ostroy 1984), als Fähigkeit neu auftretende Chancen überhaupt nutzen zu können, hat weniger mit den Kosten und der jeweiligen Form der Kurven zu tun, als mit der Fähigkeit Chancen zu erkennen, sie umzusetzen, und sich durch die Einzigartigkeit des eigenen Angebots einen Preissetzungsspielraum zu schaffen. ${ }^{21}$ Es sollte keiner besonderen Argumentation bedürfen, dass Flexibilität II auf längere Sicht weitaus größere Chancen eröffnet.

Flexibilisierung (Flexibilität I) in der Form des Verzichts auf längerfristige Konzeptionen, des Verzichts auf den Aufbau von firmenspezifischem Humankapital und dessen Sicherung durch längerfristige Verträge, oder die ausschließliche Orientierung an den Wünschen der Kunden statt autonomer Entwicklung, sind somit Strategien, die die Komplexität nicht berücksichtigen können. Sie werden leicht zur Falle des short-terminism, dem Abstellen auf bloß kurzfristigen Erfolg. Komplexität birgt die Gefahr eines short-terminism in besonderem Maße, weil in komplexen Situationen gute Indikatoren für den Erfolg, für echtes 'performance measurement' fehlen. ${ }^{22}$ Führt der Eigentümer ('principal') das Unternehmen selbst, ist das nicht weiter problematisch: er verlässt sich auf seine "animal spirits" (Keynes); er trägt das Risiko seiner Entscheidungen selbst, und profitiert auch voll vom eventuellen Erfolg. Sofern aber 'agents' die Entscheidungen treffen, Manager oder die Anlageverantwortlichen in den Fonds, sind sie zur Maximierung des Erfolgs in der kurzen Frist gezwungen, weil es keinen Indikator für den langfristigen Erfolg gibt. ${ }^{23}$ Der durch Delegation der Entscheidung auf Angestellte erzwungene Verzicht auf die lange Sicht verstellt den Blick auf die wahren Chancen des Unternehmens und verhindert die längerfristig effizienten Strategien.

21 Formell ist das zwar auch monopolistische Preissetzung; materiell unterscheidet es sich jedoch insofern, als die Konsumenten in diesem Fall zwar Substitutionsmöglichkeiten haben, jedoch bereit sind, für die Produktdifferenzierung zu zahlen.

22 Entgegen zahlreich behaupteter Informationseffizienz der Aktienmärkte zeigen nicht bloß die überschießende Entwicklung der letzen Jahre sondern auch systematische Untersuchungen, daß die Anleger einen kurzen Zeithorizont haben (Miles 1993).

23 Diese Problematik der Kapitalmarktfinanzierung durch Fonds wird auch der kontinentaleuropäischen Finanzbranche allmählich bewußt: „Ob die Orientierung an Quartalsergebnissen wirklich sinnvoll ist und die Unternehmen noch zielgerichtet und strategisch arbeiten läßt, wage ich immer mehr zu bezweifeln. ... Ich war immer besorgt, dass sich die amerikanischen Unternehmen zu sehr von Quartal zu Quartal ausrichten - gerade heute wo ein Unternehmen immense Investitionen in die Technik bewältigen muß.“ (M. Kohlhaussen, Vorstandschef der Commerzbank in Die Zeit 11, 8.3.2001, 28). 


\section{Ansatzpunkte einer Strategie des Umgangs mit Komplexität}

Eine Rückverlagerung der Entscheidung aller wichtigen Fragen der Unternehmensführung an den Eigentümer ist allerdings aus zumindest zwei Gründen verschlossen: Wegen der Komplexität der Probleme, die die Kapazität eines Einzelnen überfordern, wie wegen der breiten Streuung des Vermögens in einer demokratischen Gesellschaft mit breiter Ersparnisbildung. Wie aber kann dann der short-terminism der Flexibilisierungsideologie in der komplexen Welt der Informationsgesellschaft überwunden werden?

Eines der inzwischen breit angewandten, Teilerfolge versprechenden Strategieelemente ist die Konzentration auf die Kernkompetenzen, die 'specific assets' 24 der Firma. Damit ist zwangsläufig nicht bloß Reduktion der Komplexität verbunden, sondern auch die Chance, die Kernkompetenzen durch Spezialisierung weiter zu vertiefen. Allerdings ist dafür ein Preis zu zahlen: Einerseits droht Veralterung dieser Spezialisierung, und die Gefahr eines 'lock in' in nicht mehr marktgängige 'specific assets'; andererseits droht das ,,potentielle ,Aushöhlen“ des Unternehmens, bei dem es zu einem zerbrechlichen Gerüst reduziert wird. Denn durch pauschales Outsourcing werden sämtliche mit der Tätigkeit zusammenhängenden internen Kompetenzen und Fähigkeiten und alles Gelernte abgetreten.“ (Mogensen und Thumm, 2000, 39). Offen bleibt auch, wie die Firma Kernkompetenzen entwickelt, die anpassungsfähig und damit zukunftssicher sind. Der Ansatz bedarf daher zumindest ergänzender Strategieelemente.

Ausgangspunkt für den strategischen Umgang mit Komplexität muss eine systemtheoretische Perspektive sein, "a shift of mind - seeing interrelationships rather than linear cause-effect chains, and seeing processes of change rather than snapshots." (Senge 1990, 73). In einer komplexen Welt muß eine Überlebensstrategie an die Stelle der Optimierungsstrategie treten: "Imitating the strategy of successfully ... resilient systems, then, may not wring out the last ounce of 'efficiency' or attain the acme of specialisation that might be optimal in a surprise-free world. But in a world of uncertainty, imperfect knowledge, and constant change, such 'efficiency' strategy wins an even richer price minimizing unexpected and disastrous consequences which can arise when the causal structure of a real system turns out to be qualitatively different than expected." (Lovins and Lovins 1982, 188, zitiert nach Grabherr o.J.). Überleben in einer komplexen Welt erfordert Flexibilität II, die "possibility to move to various second period positions" als Fähigkeit neu auftretende Chancen überhaupt nutzen zu können; Elastizität, Anpassungsfähigkeit, aktives Streben nach Veränderung und organisatorisches Lernen sind dafür zentrale Voraussetzungen.

\section{I Elastizität und Anpassungsfähigkeit als institutionelle Voraussetzungen}

Elastizität muss sich sowohl auf die Strategien als auch auf die Struktur der Firma beziehen. Die Strategie muss elastisch sein, damit die Firma auf Schocks rasch und richtig reagieren kann; die Struktur muss elastisch sein, damit die Schwäche oder der Ausfall eines Elements entsprechend leicht kompensiert werden kann. Eine Möglichkeit Elastizität zu gewinnen ist Redundanz (Grabherr o. J.). In der Informationstheorie bedeutet Redundanz Ineffizienz: "Zero redundancy consti-

24 Zum Begriff der asset specifity siehe etwa Milgrom and Roberts 1992, 307. 
tuted the measure of optimal efficiency." (Landau 1969, 348), und dasselbe gilt auch für die traditionelle Unternehmenstheorie: „Alle strukturierenden Regelungen sind danach zu beurteilen, inwieweit sie mit einem Minimum an eingesetzten Gütern (Sachgüter, Arbeits- und Dienstleistungen etc.) der Aufgabenerfüllung dienen“ (Kosiol 1962, 25), also jedwede Redundanz vermeiden. In komplexen Systemen hingegen verringert Redundanz der Information, der Strukturen, der Funktionen oder der Beziehungen die Fehleranfälligkeit und eröffnet Alternativen, wenn unvorhergesehene Ereignisse den zunächst gewählten Weg nicht genügend aussichtsreich erscheinen lassen. Vor allem in Systemen, die aus wenig verlässlichen Teilsystemen bestehen, sichert sie ein hohes Maß an Verlässlichkeit; die Wahrscheinlichkeit eines Fehlers fällt mit zunehmender Redundanz exponentiell (Landau 1969, 350).

Parallel-oder Reserve-Systeme sind eine spezifische Form der Redundanz, die primär der Funktionssicherheit dienen; sie können aber auch zur Überlebenssicherung des Systems eingesetzt werden, etwa in Form der (vielfach verpönten ${ }^{25}$ ) Parallel- oder Doppelentwicklungen: Werden zwei oder mehrere Teams auf dasselbe Problem angesetzt, wirkt nicht bloß die Konkurrenz stimulierend, auch die Wahrscheinlichkeit unterschiedlicher Ergebnisse ist relativ groß; die Wahlmöglichkeiten des Auftraggebers und die Elastizität steigen, das Risiko, dass der Pfad der lokalen Maximierung in einer Sackgasse endet, wird reduziert (Simon 1983, 66ff). Weitere Möglichkeiten einer Vergrößerung der Elastizität liegen in laufender Fehlerkontrolle und Monitoring, in der Dezentralisierung von Entscheidungen und Prozessen, wie in Job rotation, die die Mitarbeiter flexibel verwendbar macht. Gut funktionierende, langfristig konzipierte Netzwerke mit Zulieferern, Vertriebspartnern und Servicefirmen können weitere Komponenten elastischer Reaktion bilden.

Elastizität als eher statisches Konzept mag noch gewisse Ähnlichkeit mit den Flexibilisierungsstrategien im Sinn von Flexibilität I haben, wenn auch Redundanz oder Parallelsysteme infolge ihrer Kosten bei Rationalisierungsexperten keineswegs hoch im Kurs stehen; Anpassungsfähigkeit, das zweite wichtige Strategieelement in einer komplexen Welt, hingegen steht in diametralem Gegensatz zu den meisten betriebswirtschaftlichen Standardstrategien. Die zentralen Ideen hinter dem Begriff Anpassungsfähigkeit sind Diversifizierung und Umgang mit Vielfalt bzw. Verschiedenheit, Konzepte die die Voraussetzungen dafür schaffen, bei Änderungen des Marktes nicht bloß eine Alternative zur Verfügung zu haben, sondern aus einer Ideenvielfalt wählen zu können. Das wiederum ist nur möglich, wenn Redundanz toleriert und Parallelentwicklungen nicht bloß zugelassen sondern vielmehr forciert werden. Flache Hierarchien, modularer Aufbau der Firma, dezentrale Entscheidungen, lokale Kontrolle und kritische Auswertung der Erfahrungen ('after action reviews') sowie Förderung der horizontalen Zusammenarbeit sind wichtige organisatorische Voraussetzungen für eine entsprechende Anpassungsfähigkeit der Organisation.

Noch eine Stufe weiter als das eher passive Konzept der Anpassungsfähigkeit geht die Strategie der bewußten Suche nach Veränderung. Sie hat zwei Komponenten: Zunächst eine bewusste Distanzierung von der jeweils gängigen Praxis: “... one reason adaptation may preclude adaptability is that people remember only those practices that are currently useful. Memory may preclude innovation." (Weick 1979). Darüber hinaus müssen kooperationsfördernde Institutionen geschaffen werden: "In particular corporate structures and organizational mechanisms must be consciously developed to promote particular 'sociotechnical alignments' and facilitate 'knowledge integration' between specialist divisions inside the firm and between allied companies across different industrial sectors." (Collinson and Molina 1998, 77). Veränderung muss aktiv gesucht werden, da bestehende Firmen zumeist in ihrer Normaltätigkeit gefangen sind: ${ }^{26}$ Markante Neuerungen erwerben sie zumeist durch Akquisition von dynamischen jungen Firmen, einschneidende Reorganisationsmaß-

25 Siehe etwa die Bemühungen der EU durch Koordinierung der Forschung zu vermeiden, daß in zwei Staaten an demselben Problem gearbeitet wird - ein typischer Versuch Redundanz zulasten kurzfristiger Effizienz $\mathrm{zu}$ verringern.

26 Wirklich neue Konzepte, Verfahren oder Produkte kommen in der Regel von neuen Firmen. 
nahmen oder Änderungen der Technologie setzen zumeist negative Schocks voraus (Cooper and Haltiwanger 1993; Aguirregabiria and Alonso-Borrego 2001). Um endogen änderungsfähig zu sein, müssen Firmen unkonventionelle Nebenaktivitäten ('active fringe') zulassen - neue Ideen kommen zumeist von außen, durch Kontakte mit Personen aus anderen Branchen, mit anderen Auffassungen und Konzepten (Noteboom 1999). Die Firmen müssen Fähigkeit wie Bereitschaft entwickeln, sich mit den Ideen der unkonventionellen ,Außenseiter in der Firma' auseinanderzusetzen, passende aufzugreifen und umzusetzen. Mit einer strikten Strategie der Konzentration auf die Kernaktivitäten ist das nicht kompatibel.

\subsection{Corporate Vision und organisatorisches Lernen als Instrumente}

Der Rahmen der institutionellen Voraussetzungen zur Erreichung von Flexibilität II muß durch konkretes Handeln der Firma aufgefüllt werden, um Beziehungsnetzwerke aufzubauen und die Prozesse zu erkennen, die die künftige Entwicklung bestimmen. Ausgangspunkt dafür muß eine Corporate vision sein, eine Vorstellung über Form, Position und Aufgaben der Firma in der Zukunft. Das widerspricht nicht der grundsätzlichen Unprognostizierbarkeit komplexer Systeme, die keineswegs bedeutet, dass sich über die künftige Entwicklung gar nichts aussagen lässt; allerdings kann es sich nicht um determinististische Prognosen ('forecast') handeln, sondern um 'foresight', um die probabilistische Einschätzung künftiger Entwicklungen, um ,Zukunftslandkarten', in die jederzeit neue Routen eingezeichnet werden können; ${ }^{27}$ sie sind als Basis des organisatorischen Lernens der Firma unverzichtbar. Dafür stehen verschiedene Instrumente zur Verfügung, die, je nach den Voraussetzungen, isoliert oder - besser - in Kombination eingesetzt werden können: Modellierung und Simulation, mit denen das Verhalten und die Dynamik des jeweiligen (komplexen) Systems erkennbar gemacht werden kann, Verfahren der Mustererkennung, aber auch Experimente, mit denen die Reaktionen des Systems herausgefunden werden können. Entscheidend sind weniger die Ergebnisse solcher ,foresight-Übungen“, als vielmehr das organisatorische Lernen, die systematische Beschäftigung möglichst vieler Beteiligter mit unterschiedlichen längerfristigen Entwicklungspotentialen; dadurch bekommt ein breiter Kreis von Mitarbeitern ein Gefühl für die komplexe Entwicklung, in die die Firma eingebettet ist, für deren Dynamik und für mögliche Alternativen.

Im Zentrum von Überlegungen betreffend Maßnahmen zur Erhöhung der Flexibilität II aus Firmensicht sollte die capability-Theorie der Firma stehen, die diese als ein Bündel spezifischer Fähigkeiten sieht: "The firm is defined by what it knows; its knowledge determines what it can do and how." (Metcalfe and de Liso 1998, 19). Diese spezifischen Fähigkeiten muss die Firma als 'strategic assets' gezielt aufbauen, um mit der Unsicherheit der Realität zurecht zu kommen. Die capabilities mögen zum geringen Teil in Patenten und Firmenhandbüchern kodifiziert sein, überwiegend bestehen sie aus dem Wissen der Mitarbeiter, und vielfach handelt es sich um 'tacit knowledge', Wissen das nicht ohne weiteres kodifiziert und weitergegeben werden kann. Die Mitarbeiter mit ihrem individuellen Wissen bilden im Rahmen der Organisation der Firma und mit Hilfe komplementärer, vielfach firmenspezifischer Produktionsfaktoren (Teece 1987) die capability der Firma als einen "competence pool” (McKelvey 1982).

Komplexität und Verflechtung erfordern, dass an die Stelle der Strategie des 'planned change' der siebziger Jahre eine Strategie des 'managed learning' und der Förderung der Kreativität treten muss. "It's just not possible any longer to 'figure it out' from the top"; und daher unverzichtbar, "to

27 Siehe dazu etwa OECD 1996. 
discover how to tap people's commitment and capacity to learn at all levels." (Senge 1990, 4). Voraussetzung dafür ist 'absorptive capacity', die Fähigkeit der Firma neue Ideen aufzunehmen. "An organization's ability to learn and translate that learning into action is the ultimate competitive advantage." (J. Welch $\left.{ }^{28}\right)$.

Zum Aufbau firmenspezifischer Kompetenz - als Kombination des individuellen Humankapitals der Mitarbeiter mit komplementären firmenspezifischen Produktionsfaktoren innerhalb des Rahmens der Organisation der Firma - ist Lernen auf allen Ebenen der Organisation erforderlich. Die gezielte Bildung firmenspezifischen Humankapitals durch die Mitarbeiter muss von der Firma bewusst gestaltet werden, da für die Mitarbeiter allgemeines, firmenunspezifisches Humankapital wertvoller ist: es macht sie krisenresistenter und erhöht ihre Mobilität; sie können die Firma in Problemsituationen leichter verlassen. ${ }^{29}$ "The ability of the firm to absorb and to act upon knowledge depends in part on staff continuity. Practical steps to achieve this include on-the-job promotion and share options, or, more subtly, technology can be deployed strategically to 'lock-in' staff. Corporate vision can add as a focusing device." (Swann, 1998, 118). ${ }^{30}$ Firmeninterne Aus- und Weiterbildung der Mitarbeiter kann firmenspezifisches Wissen in besonderem Maße vermitteln und eine besonders enge Firmenbindung schaffen. ${ }^{31}$ Lernen muß ein kontinuierlicher Prozeß sein, und neben individuellem Lernen im Sinne des Erwerbs firmenspezifischen Wissens auch kollektive Formen umfassen - individuelles Lernen kann organisatorisches nicht ersetzen. Zunehmend werden auch spirituelle Aspekte für das Lernen und Verändern von Organisationen betont. Das Wissen sollte schon in seinem Entstehen erfasst, und nicht bloß bestehendes Wissen mitgeteilt werden. Unkonventionelle Lernformen, bis hin zur Auseinandersetzung mit Kunst, können dazu beitragen (Glasl 2000, 2).

Hire and fire-Strategien wirken dem Erwerb firmenspezifischen Humankapitals ebenso entgegen ${ }^{32}$ wie Verunsicherung der Mitarbeiter durch häufigen Strategiewechsel der Firma als Folge häufig wechselnder Manager oder Eigentümer. Bloß temporäre Verträge können besonders kontraproduktiv wirken, da die temporär Beschäftigten daran interessiert sein werden, gerade die strategischen Kompetenzen der Firma kennenzulernen, deren Kenntnis ihre Beschäftigungschancen in Konkurrenzfirmen verbessert.

Dieselben Argumente wie für das Personal gelten auch für längerfristige Vertragsbindungen gegenüber Zulieferern oder eigenständigen Vertriebsunternehmen. Je kürzer diese Bindungen, je leichter kündbar, desto mehr müssen die Zulieferer auf breite Verwendbarkeit ihrer Investitionen und ihrer Entwicklungsaufwendungen zu achten, und desto weniger werden sie bereit sein für ihren (vermutlich bloß temporären) Kunden spezifische Varianten zu entwickeln, womit dessen Chance sinkt, sich mit seiner Produktionspalette von den Konkurrenten markant abzuheben. Dasselbe gilt mutatis mutandis für die Dauer der Bindung an eigenständige Vertriebsunternehmen.

28 CEO von General Electric.

29 Die Flexibilisierungsstrategie der bloß temporären Beschäftigung erzieht egoistische Mitarbeiter; nur Mitarbeiter, die sich mit der Firma identifizieren, eine langfristige Firmenbindung anstreben und in der Firma Karriere machen wollen, werden bereit sein, firmenspezifisches Humankapital zu erwerben

30 Auch Aktienoptionen bieten bloß eine mittelfristige Perspektive, und legen den Managern gerade vor ihrer Fälligkeit kurzfristig kurssteigernde Aktivitäten besonders nahe.

31 Flexibilisierung im Sinn von Flexibilität I, die Fähigkeit zu rascher Reaktion, mag zwar das Offenhalten zahlreicher Alternativen beinhalten; solche Alternativen zu entwickeln, bereit zu halten, und bei Bedarf umzusetzen, erfordert aber firmenspezifische Kompetenz, Fähigkeiten, über die die Konkurrenten nicht verfügen; durch ad hoc-Zukauf von Experten, Expertise und Vorleistungen kann solche firmenspezifische Kompetenz vielleicht erweitert, niemals jedoch geschaffen werden. Flexibilisierungsstrategien im Sinne einer Vergrößerung der Flexibilität I sind insoweit Scheinlösungen und wirken längerfristig sogar kontraproduktiv, da sie dem Aufbau firmenspezifischer Kompetenz entgegenwirken.

32 Das gilt in besonderem Maße für Europa; in den USA hingegen erfolgen die Freisetzungen vielfach bloß temporär, d. h. dass die Freigesetzten bei Änderung der Lage bevorzugt wieder eingestellt werden. 


\section{Ausblick}

Der Capability-Ansatz zeigt, dass der Verzicht auf Flexibilisierung im Sinne eines Verzichts auf kurzfristige Reaktionsmöglichkeiten (Flexibilität I) zugunsten langfristiger Personalpolitik und Firmenbindung dem Aufbau firmenspezifischer Kompetenz ganz erheblich nützen kann; kurzfristige Verträge vermeiden zwar Bindungen, ermöglichen aber nicht die Entwicklung von Alternativen zu den jeweiligen Strategien. Mehr noch als auf Firmenebene ist Flexibilisierung jedoch auf volkswirtschaftlicher Ebene problematisch. Über die Verringerung der firmenspezifischen Kompetenzen erschwert sie den volkswirtschaftlichen Strukturwandel; mangels langfristiger Personalbindung wird nicht bloß die Ausbildung durch die Firmen reduziert, es werden auch die individuellen Anreize zur Aus- und Weiterbildung durch Verunsicherung und kurzfristige Orientierung geschwächt; Fachkräftemangel ist die unvermeidliche - und bereits sichtbare - Folge. Dazu kommt, dass die rasch alternde Bevölkerung risikoscheuer und weniger lernfähig wird. Diejenigen Firmen, die sich schon jetzt auf die Änderung der Voraussetzungen einstellen, die Ausbildung ihrer Spezialisten selbst in die Hand nehmen und wieder Instrumente entwickeln, um eine Stammbelegschaft mit firmenspezifischem Wissen aufzubauen und längerfristig zu halten, werden einen nicht unerheblichen Konkurrenzvorsprung erlangen. Eine Wirtschaftspolitik, die diese Tendenzen vorwegnimmt, bewusst macht, und die entsprechenden Rahmenbedingungen schafft, wird zur Sicherung der Konkurrenzfähigkeit und Standortqualität der betreffenden Landes erheblich beitragen.

\section{Literatur}

AGUIRREGABIRIA, V. and C. ALONSO-BORREGO (2001): Occupational structure, technological innovation, and reorganization of production, Labour Economics 8, 43-73.

CHRISTENSEN, C. M. and R. S. ROSENBLOOM (1995): Explaining the attacker's advantage: technological paradigms, organizational dynamics, and the value network, Research Policy $24,223-257$.

CHRISTENSEN, C. M. (1997): The innovator's dilemma: When technologies cause great firms to fail, Harvard Bus.School Press, Boston.

COLLINSON, S. and A. MOLINA (1998): Reorganizing for knowledge integration and constituency building: product development at Sony and Philips, in R. Coombs et al (eds), Technological change and organization, Elgar, Cheltenham/Northampton, MA., 76-107.

COOPER, R. and J. HALTIWANGER (1993): The aggregate implications of machine replacement: theory and evidence, American Economic Review 83, 360-382.

Föhrenbergkreis (1997): Jobshift. Überlegungen zur neuen Welt der Arbeit, IWIP Institut für Arbeit und Politik, Wien.

GLASL, F. (2000): Was wir in Boston über Lernen lernen konnten, Trigon Themen 4/00, 2.

GORDON, R. J. (2000): Does the ,New Economy' measure up to the great inventions of the past? NBER-Working Paper No.W7833.

GRABHERR, G. (o. J.): Zur Ökonomie des Überflusses: Die Bedeutung von Redundanz für die Regionalentwicklung, Manuskript, Wissenschaftszentrum Berlin. 
HAMMERMESH, D. (1993): Labor demand, Princeton University Press, Princeton NJ.

HANDY, Ch., et al (1997): Rethinking the future. Business, principles, competition, control, leadership, markets and the world, N. Brealey, London.

HOLMSTROM, B. R. and J. TIROLE (1989): The theory of the firm, in R. Schmalensee and R. D. Willig, eds, Handbook of industrial organization, Elsevier, Amsterdam, Vol I, 63-133.

JONES, R. A. and J. M. OSTROY (1984): Flexibility and uncertainty, Review of Economic Studies $51,13-32$.

KLEIN, B. H. (1984): Prices, wages, and the business cycle: A dynamic theory, Pergamon, New York.

KOSIOL, E. (1962): Organisation der Unternehmung, Gabler, Wiesbaden.

LANDAU, M. (1969): Redundancy, rationality, and the problem of duplication and overlap, Public Administration Review 29, 346-358.

LANDES, D. (1999): Wohlstand und Armut der Nationen, Siedler, Berlin.

LOVINS, A. B. and L. H. LOVINS (1988): Brittle power. Energy strategy for national security, Andover.

LUHMANN, N. (1988): Soziale Systeme. Grundriß einer allgemeinen Theorie, Suhrkamp, Frankfurt.

MALERBA, F. et al (1997): Persistence of innovative activities, sectoral patterns of innovation and international technological specialization, International Journal of Industrial Economics 15(6), 801-826.

McKELVEY, W. (1982): Organizational Systematics, University of California Press, Berkeley.

METCALFE, St. and N. de LISO (1998): Innovation, capabilities and knowledge: the epistemic connection, in R. Coombs et al, (eds), Technological change and organization, Elgar, Cheltenham/Northampton, MA., 8-27.

MILES, D. (1993): Testing for short terminism in the UK stock market, Economic Journal 102, $1.379-1.396$.

MILGROM, P. and J. ROBERTS (1992): Economics, organization \& management, Prentice Hall, Upper Saddle River, NJ.

MOGENSEN, J. und N. THUMM (2000): Outsourcing von Geschäftsdienstleistungen, IPTS Report 49, 36-44.

NOTEBOOM, B. (1999): Innovation and inter-firm linkages: new implications for policy, Research Policy 28, 793-805.

OECD (1996): STI Review 17: Special issue on Government Technology Foresight Exercises, OECD, Paris.

PETERS, T. (1994): Tom Peter's seminar - Crazy times call for crazy organisations, MacMillan, London.

Sachverständigenrat zur Begutachtung der gesamtwirtschaftlichen Entwicklung (2000): Chancen auf einen höheren Wachstumspfad. Jahresgutachten 2000/01, Metzler-Poeschel, Stuttgart.

SCARPETTA, St. et al (2000): Economic growth in the OECD area: Recent trends at the aggregate and sectoral level, OECD Working Papers 248, OECD, Paris.

SCHMIDT, R. H. und J. MAßMANN (1999): Drei Mißverständnisse zum Thema 'Shareholder Value', in Kumar, B. et al, Hg, Unternehmensethik und die Transformation des Wettbewerbs, Schäffer/Poeschel, Stuttgart, 125-151. 
SCHWEIKHART, N. (2001): Der getriebene Chef, Die Zeit 20, 10.5.2001, 26.

SENGE, P. M. (1990): The fifth discipline: The art and practice of the learning organization, Doubleday, New York.

SHAPIRO, C. and H. VARIAN (1998): Information rules. A strategic guide to the network economy, Harvard Business School, Boston, http://www.inforules.com.

SIMON, H. (1983): Reason in human affairs, Blackwell, Oxford.

STIGLER, G. (1939): Production and distribution in the short run, Journal of Political Economy 47, 305-327.

SWANN, P. (1998): Rapid technological change and shortening business horizons, in R. Coombs et al, eds, Technological change and organization, Elgar, Cheltenham/Northampton, MA., $108-123$.

TEECE, D. J. (1987): Capturing value from technological innovation: Integration, strategic patterning and licensing decisions, in Brooks, H. and B. R. Guide, eds, Technological and global industry, National Academy Press, Washington DC.

TICHY, G. (2001): Das Nutzer-Paradoxon und seine Bedeutung für die europäische Innovationsschwäche. Neue Ansatzpunkte für die Technologiepolitik? in W. Fuchs und O. Horvath, Hg, Wirtschaftsstandort Österreich. Von der Theorie zur Praxis, Bundesministerium für Wirtschaft und Arbeit, Wien, 207-230.

TRISOGLIO, A. (1995): Complexity: The challenges, presented at the workshop 'Risk, Policy and Complexity, mimeo, IIASA.

WEICK, K. E. (1979): The social psychology of organizing, Addinson-Wesley, Reading, Mass. 


\section{Bisher erschienene manu:scripte}

ITA-0I-0I Gunther Tichy, Walter Peissl (12/2001): Beeinträchtigung der Privatsphäre in der Informationsgesellschaft. <http://www.oeaw.ac.at/ita/pdf/ita_01_01.pdf>

ITA-0I-02 Georg Aichholzer(12/2001): Delphi Austria: An Example of Tailoring Foresight to the Needs of a Small Country. <http://www.oeaw.ac.at/ita/pdf/ita_01_02.pdf>

ITA-0I-03 Helge Torgersen, Jürgen Hampel (12/2001): The Gate-Resonance Model: The Interface of Policy, Media and the Public in Technology Conflicts.

$<$ http://www.oeaw.ac.at/ita/pdf/ita_01_03.pdf>

ITA-02-0I Georg Aichholzer (01/2002): Das ExpertInnen-Delphi: Methodische Grundlagen und Anwendungsfeld ,Technology Foresight“.

$<$ http://www.oeaw.ac.at/ita/pdf/ita_02_01.pdf>

ITA-02-02 Walter Peissl (01/2002): Surveillance and Security - A Dodgy Relationship. $<$ http://www.oeaw.ac.at/ita/pdf/ita_02_02.pdf>

ITA-02-03 Gunther Tichy (02/2003): Informationsgesellschaft und flexiblere Arbeitsmärkte. <http://www.oeaw.ac.at/ita/pdf/ita_02_03.pdf> 